\title{
EDITORIAL
}

\section{¿DEBEN ESTAR LAS TÉCNICAS DE CONSENSO INCLUIDAS ENTRE LAS TÉCNICAS DE INVESTIGACIÓN CUALITATIVA?}

\author{
Cristina Pérez Andrés
}

Subdirección General de Promoción de la Salud y Epidemiología. Dirección General de salud Pública y Consumo. Ministerio de Sanidad y Consumo.

En los últimos tiempos vienen publicándose en las revistas del ámbito de las ciencias de la salud, tanto nacionales como internacionales ${ }^{1-3}$, trabajos sobre la metodología cualitativa que incluyen entre sus técnicas las llamadas técnicas de consenso. Lo mismo ocurre en los programas de los cursos de salud pública, así como en las clasificaciones que de las metodologías de investigación hacen los tratados u otros textos sobre salud pública ${ }^{4}$. Así, se incluyen en la metodología cualitativa técnicas como el grupo Delphi, el grupo nominal, el brainstorming, etcétera. Sin embargo, no ocurre lo mismo en los textos que sobre metodología cualitativa escriben los especialistas en sociología u otras disciplinas diferentes a la salud pública ${ }^{5-8}$. Tampoco han incluido las técnicas de consenso como pertenecientes a la metodología cualitativa la serie de artículos que sobre ella ha publicado recientemente JAMA $^{9-11}$, que sólo contempla como técnicas cualitativas la observación participante, la entrevista abierta y los grupos de discusión. Esta situación produce un nivel de confusión que no estaría mal aclarar; es lo que intento al escribir este editorial.

Dentro de las técnicas para el cstudio de la realidad social, de la que forman parte los fenómenos relativos a la salud y a la enfermedad, distinguimos entre las llamadas cuantitativas o distributivas y las cualitativas o estructurales. Las primeras describen la realidad a través de una serie de variables con las que se construyen cuestionarios cuyas respuestas sirven para calcular la distribución de la frecuen- cia de las mismas en las personas que forman la muestra. Cuando se trata de muestras extraídas de la población, estos resultados se pueden inferir al resto de la misma utilizando diferentes cálculos numéricos. Las segundas, que por oposición a éstas, se denominan cualitativas o estructurales, tratan de encontrar la estructura de las relaciones que hacen comprensible el sentido de las representaciones sociales en el lenguaje de los sujetos que forman la sociedad. En concreto, en el ámbito de la salud, la metodología cualitativa la explora desde el punto de vista de la concepción cultural y simbólica que la sociedad tiene de la misma ${ }^{12}$.

Lo fundamental de la metodología cualitativa o estructural es que trabaja con el análisis e interpretación del lenguaje (verbal o no verbal) obtenido en condiciones de investigación, siendo las más características la observación participante, las historias de vida, la entrevista abierta y el grupo de discusión ${ }^{5-11}$. Sus marcos de referencia son la linguística, la semántica (estudio del sentido de los significantes linguiisticos), la antropología, el psicoanálisis, la sociología no cuantitativa y la hermeneutica ${ }^{5-11}$. En la metodología cualitativa el elemento de estudio no son los hechos sino los discursos, su herramienta el análisis y la interpretación del lenguaje ya que lo que busca es determinar la significación de los fenómenos sociales para comprender su sentido. En la metodología cualitativa no se usan cuestionarios, ni abiertos ni cerrados, porque no se trata de que los sujetos que participan en la investigación respondan, sino de que hablen. Además, en las técnicas 
cualitativas el investigador nunca ofrece su opinión a las personas que participan en la investigación, con el fin de no producir influencias que modificarían los resultados; y la autoridad que el investigador tiene sobre los sujetos que participan en la investigación es de carácter simbólico y no reconocida previamente por ellos. De hecho nunca debería moderar una técnica cualitativa una persona con ascendencia profesional o de otro tipo sobre los participantes en la misma.

Las técnicas de consenso, aunque no llevan un diseño muestral estricto en cuanto a la selección de las personas que han de formar parte de la muestra (son elegidas sin que intervenga el azar) ni en la determinación del tamaño de la misma (no es representativo numéricamente de la comunidad de profesionales que podrían opinar), utilizan cuestionarios para la recogida de la información y la técnica cuantitativa a través de medidas estadísticas de agregación para obtener sus resultados. En realidad lo que persiguen estas técnicas es obtener el grado de consenso o acuerdo de los especialistas sobre el problema planteado, utilizando los resultados de investigaciones anteriores, en lugar de dejar la decisión a un solo profesional ${ }^{3}$.

La primera que se utilizó de ellas fue la técnica Delphi ${ }^{3,13-15}$, creada en 1952 por Ia Rand Corporation de Estados Unidos. Aunque se dice que utiliza un proceso de grupo los miembros del mismo nunca se llegan a reunir, es más, cada participante desconoce quienes son las otras personas que participan con él, por lo que el grupo en realidad no existe. Además se vale de una serie de cuestionarios que se envían por correo a los participantes, con el fin de que sobre el mismo se elijan las respuestas que se crean más convenientes, para así reducir la incertidumbre sobre el problema planteado. Con el análisis cuantitativo de las respuestas del primer cuestionario se elabora un segundo cuyas opciones son votadas por los participantes; con un proceso similar al seguido con el primer cuestionario, se construye un tercero para obtencr cl voto final de los participantes con los que se elabora el informe que, una vez más, precisa de la cuantificación para su realización.

El grupo nominal (ol panel de expertos) ${ }^{3,16}$, desarrollado en 1968, es una variante de la téc- nica Delphi y su nombre procede de que los miembros del grupo sí saben quienes son los que están participando junto con él, (conocen su nombre), al contrario que en el Delphi, en el que permanecen anónimos. En el grupo nominal aunque las personas del grupo sí se reúnen, en un principio no se pueden comunicar verbalmente y el coordinador del grupo debe ser una persona con autoridad reconocida sobre el tema a tratar. En la primera parte del proceso cada participante anota individualmente las ideas que le surjan sobre la pregunta realizada por el coordinador; después hay una exposición exhaustiva de todas las ideas generadas, una discusión sobre las mismas y una votación individual y anónima por los miembros del grupo, según una escala cuantitativa previamente establecida, de cero a cien, de uno a cinco,... Tras la obtención de los resultados estadísticos de las puntuaciones dadas a las preguntas del cuestionario, se someten a posterior discusión los puntos más conflictivos. $\Lambda 1$ final de esta reunión se vuelven a puntuar las propuestas, definiéndose como consensuadas por el panel las más votadas. En cada respuesta se emplea el valor de la mediana o de otros percentiles para medir la tendencia central de las puntuaciones a cada pregunta y las medias aritméticas del acuerdo y desacuerdo, previamente definidos numéricamente.

En la técnica brainstorming ${ }^{4,17}$ (tormenta de ideas) el líder puede presentar sus propias ideas para estimular la participación de los componentes del grupo. El conjunto de ideas producidas representa puntos de partida que, tras un ejercicio de reestructuración, da lugar a una lista abreviada sobre las que se eligen por puntuación las mejores ideas.

En definitiva, las técnicas a las que nos hemos referido utilizan herramientas cuantitativas que la metodología cualitativa no precisa, como son el cuestionario y la cuantificación, así como la intervención de los investigadores con sus propias opiniones en la producción de las de los participantes.

Pero la principal diferencia entre las técnicas de consenso y las técnicas cualitativas es el tipo de lenguaje con el que ambas trabajan $^{18}$. Los participantes en las técnicas de consenso utilizan el lenguaje científico-técnico, 
en el cual los códigos utilizados son monosémicos (a un significante le corresponde un solo significado) y denotativos (significados concebidos objetivamente), y la función del lenguaje que prevalece, según el esquema de Jakobson, es la referencial (el mensaje se refiere directamente al objeto, luego es objetivo) y por lo tanto en él no hay nada que interpretar. Al contrario, las técnicas propiamente cualitativas trabajan con el lenguaje social tradicional, cuya función no es sólo referencial sino que incluye la función emotiva (relación entre el mensaje y el emisor), connotativa (relaciones entre el mensaje y el receptor), estética (el objeto de la comunicación es el propio mensaje), fática y metalingüística (el mensaje tiene por objeto otro mensaje), cuyos códigos son polisémicos (un significante puede remitir a varios significados) y connotativos (conlleva valores subjetivos atribuidos al significante), en los que las representaciones sociales y metáforas constituyen el material que el investigador analiza e interpreta, no para cuantificarlo sino para encontrar el sentido oculto que contienen (hermeneútica). Mientras que el saber científico utiliza una codificación por homología (los significantes mantienen entre sí la misma relación que los significados), el saber tradicional se basa en sistemas en los cuales el significante mantiene una relación de analogía (metafórica) con el significado. $\Lambda$ sí, a diferencia de las técnicas de consenso, la metodología estructural o cualitativa, no sólo no utiliza nunca cuestionarios ni cuantifica las respuestas, sino que analiza e interpreta el lenguaje para encontrar el sentido oculto del fenómeno social que se está investigando, herramienta que no utilizan las técnicas de consenso.

Ello no quiere decir que con los profesionales sanitarios no se puedan hacer investigaciones con metodología cualitativa. De hecho existen numerosos ejemplos de investigaciones realizadas con grupos de discusión en los que los participantes eran médicos, enfermeros $y$ gestores ${ }^{19-21}$. Pero su objetivo no era buscar su consenso sobre un problema técnico, sino conocer las vivencias, opiniones y representaciones de los participantes en los grupos sobre los temas investigados y el por qué de las mismas. No era su conocimiento científico el que interesaba en la investigación, sino su discurso social sobre los temas planteados.

En resumen, dos son los argumentos que van en contra de clasificar las técnicas de consenso entre las correspondientes a la metodología cualitativa:

- El tipo de lenguaje con el que se trabaja en cada una de ellas: científicotécnico (referencial, monosémico y denotativo) en las técnicas de consenso $y$, por tanto, no interpretable; y tradicional (emotivo, estético,... polisémico y connotativo) en las técnicas cualitativas.

- El tipo de datos y herramientas utilizados: numéricos y cuantificables en las técnicas de consenso y discursivos e interpretables en las cualitativas.

Por último, señalar que mientras que las técnicas de la metodología cualitativa producen información primaria (original), las técnicas de consenso producen información secundaria, en el sentido de que no son resultados de investigaciones originales los que ofrecen, sino que trabaja con los datos obtenidos por otras investigaciones primarias, diseñadas, en su gran mayoría, con metodología epidemiológica.

En mi opinión, las técnicas de consenso no son ni metodología epidemiológica ni metodología cualitativa, sino simplemente eso, técnicas que buscan el consenso, el acuerdo de un conjunto, que no grupo, de personas expertas en un tema científico-técnico, para acordar cuáles son las mejores ideas o las mejores decisiones a tomar sobre un problema planteado.

Me queda por añadir que desde un principio el objetivo de este editorial ha sido llamar a la reflexión y al debate constructivo sobre unas técnicas, las de obtención de consenso, que sólo en el ámbito de la salud pública se encuentran clasificadas entre las técnicas de la metodología cualitativa, con las que la única característica que comparten es la de no tener un diseño muestral estricto. Por ello, será bienvenida toda aportación que contribuya a clasificar correctamente estas técnicas, así como el planteamiento de las dudas u opiniones en 
contra que con este editorial se hayan podido generar.

\section{BIBLIOGRAFÍA}

1. March Cerdá JC, Prieto Rodríguez MA, Hernán García M y Solas Gaspar O. Técnicas cualitativas para la investigación en salud pública y gestión de servicios de salud: algo más que otro tipo de técnicas. Gac Sanit 1999; 13:312-319.

2. Íñiguez Rueda L. Investigación y evaluación cualitativa: bases teóricas y conceptuales. Aten Primaria 1999; 23:496-502.

3. Jones J and Hunter D. Consensus methods for medical and health services research. BMJ. 1995 Aug 5;311(7001):376-80.

4. March Cerda JC, García Calvente $\mathrm{M}^{\mathrm{a}}$ del M y Prado Torres A. Los métodos de investigación y evaluación en la promoción de la salud. En: Salud Pública. Martínez Navarro F, Antó JM, Castellanos JL, Gili M, Marset P y Navarro V editores. Madrid: Mc Graw-Hiill Interamericana de España; 1997.

5. Métodos y Técnicas cualitativas de investigación en ciencias sociales. Madrid: Síntesis Psicológica; 1994.

6. Alonso LE. La mirada cualitativa en sociología. Madrid: Fundamentos; 1998.

7. El análisis de la realidad social. Métodos y Técnicas de investigación. Madrid: Alianza Universidad; 1990.

8. Schartz H y Jacobs H. Sociología cualitativa; método para la reconstrucción de la realidad. México: Editorial Trillas;

9. McAlister FA, Straus SE, Guyatt $\mathrm{GH}$ and Haynes RB. Users'Guides to the medical literature XX. Integrating Research evidence with the care of the individual patient. JAMA 2000; 283:2829-2836.
10. Giacomini MK and Cook DJ. Users'Guides to the medical literature XXIII. Qualitative Research in health care A. Are the results of the study valid? JAMA 2000; 284:357-358.

11. Giacomini MK and Cook DJ. Users'Guides to the medical literature XXIII. Qualitative Research in health care $B$. What are the results and how do they help me care for my patiens? JAMA $2000 ; 284: 478-482$.

12. Conde F y Pérez Andrés C. La investigación cualitativa en salud pública. Rev Esp Salud Pública 1995; 69: 145-149.

13. Gervas JJ y Fernández Pérez MM. La técnica Delphi. Clínica Rural 1989; 312:38-42.

14. Pyke DL. A practical approach to Delphi. Futures 1970.

15. Pill J. The Delphi method: substance, context, a critique and an annotated bibliography. Soc Econ Plan Sci 1971; 3:37-71.

16. Gervas JJ y Fernández Pérez MM. El grupo nominal, un método de consenso. Clínica Rural 1989; 313:42-50.

17. Tudor R. Técnicas de grupo basadas en los principios y la práctica del braingstorming. En: La creatividad. Análisis y solución de problemas empresariales. Bilbao: Deusto; 1977.

18. Guiraud P. La semiología. México: Siglo XXI; 1991.

19. Pérez Andrés C. Aceptación del contrato programa por el personal sanitario de la atención primaria de Madrid: un estudio cualitativo. Rev Sanid Hig Pública 1995; 69:79-88.

20. Consejería de Salud. Actitudes ante el asma. Los asmáticos y los profesionales opinan. Madrid: Consejería de Salud; 1994. Documentos técnicos de salud pública, núm 17.

21. Consejería de Salud. Discurso del personal sanitario de la Comunidad de Madrid en torno a la infección por el VIH/Sida. Madrid: Consejería de Salud; 1992. Documentos técnicos de salud pública, núm 11 . 